\title{
Paper machine dry line position control during grade changes
}

\author{
John Erik Larsson and Thomas Gustafsson \\ Control Engineering Group \\ Luleå University of Technology, S-971 87 Luleå, Sweden \\ joppe@sm.luth.se, thomas@sm.luth.se
}

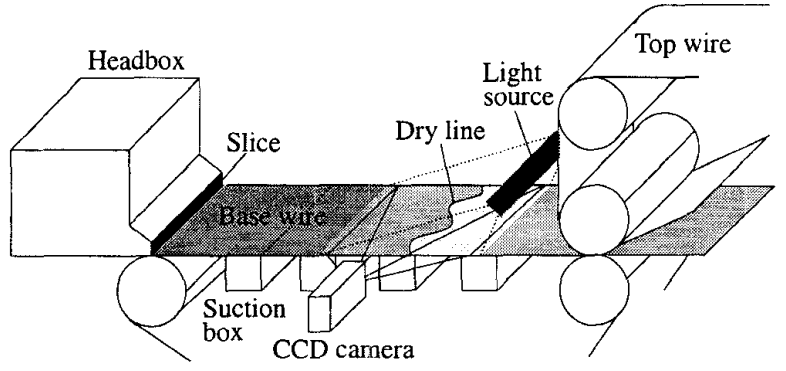

Figure 1: View of part of the wet end.

\begin{abstract}
The detection and control of the dry line machine direction position on a fourdrinier paper machine are considered in this work. The dry line position is detected using a digital image processing system consisting of a CCD camera, a frame grabber and a standard PC. The system was installed on a kraft liner machine and both open loop and closed loop experiments were performed with encouraging results. The introduction of automatic control of the dry line position reduces the time for grade changes and it also reduces the machine operators work load. The reduced time leads to smaller production losses and hence to economical benefits.
\end{abstract}

\section{Introduction}

The function of a paper machine is to remove water from a dilution of cellulose fibres and water, in such a manner that the final product possesses some desired properties. The paper machine can be divided into a wet end and a dry end. The wet end starts with the headbox which spreads the fibre dilution (stock) as even as possible over a moving endless wire, see figure 1. Approximately $95 \%$ of the water is removed through the wire by suction boxes and hydrodynamic devices and a continuous fibre felt is formed. The fibre felt leaves the $0-7803-4530-4 / 98 \$ 10.00$ (C) 1998 AACC wire and passes through a number of presses and driers, which constitute the dry end, before it is rolled onto tambours. In the transition from a dilution into a felt an optical phenomenon can be observed. The surface of the dilution is highly reflective while the surface of the felt is mat. Depending on the light and the angle of view the dilution area can be seen as a dark area and the felt area as a bright area, see figure 2. The transition from dark to bright can be seen as a line across the wire and it is called the dry line, see figure 1 . The dry line is not a straight line and its position and contour are continuously changing.

Many important properties of the paper, such as basis weight and strength, are determined in the wet end, but not measured until the end of the machine, thus leading to long time delays. This makes good over all wet end control very important. Headbox total pressure, level and concentration control is a well treated area, see [7] and [9]. Headbox outlet jet velocity is a very important factor in the forming process which affects the strength of the paper. The control of headbox outlet jet velocity is treated in [8]. The control of the cross machine direction properties are also well studied, see [4].

The position of the dry line is very important. If the dry line is positioned too far away from the headbox, too little water may have been removed so that the continuous felt doesn't have the strength needed to pass the sections succeeding the wire, resulting in costly production stops. Placing the dry line too close to the headbox reduces the strength and may lead to an uneven fibre distribution, so called flocculation. see [12]. The joining of the top and base layer may also be poor if the dry line is positioned too close to the headbox.

There are many factors affecting the position of the dry line, and their effect on the magnitude of the dry line movement is often very difficult to predict. Some of the more important factors are the headbox slice opening (affecting the volume outflow), machine speed, the negative pressure of the suction boxes and the beating degree of the fibres.

During steady state production the manual control of the dry line position is a relatively easy task for a 
skilled machine operator, with the headbox slice opening as the main actuator. The negative pressure of the suction boxes is also used but it has less effect on the dry line position. The manual control task is much more demanding during grade changes when the machine speed and other factors affecting the dry line are changed, thus needing the full attention of one operator. Automatic control of the dry line position would be welcomed by the machine operators but no such system has been available until now. Another reason for automatic control of the dry line position is to reduce the wet end disturbances caused by stepwise changes in slice opening, thus reducing the time needed for a grade change, see [13].

The position and contour of the dry line are continuously changing and the light conditions on the plant are often poor. The position and contour of the dry line are estimated by the operators by in situ visual inspection, or by remote inspection via a video image. The criterion to determine what is a dry line or not often varies between different operators. It is thus a difficult task to determine the position and contour of the dry line in a consistent manner.

Some early attempts of automatic dry line detection can be found in [1] and [2], but they were probably hampered in practice by the limited computational capacity available at the time. The availability of fast computers and video signal digitizing hardware has renewed the interest in automatic dry line detection, see [5], [6] and [10]. The work in [10] is based on [11] and is mainly concerned with the detection of, and the possibility to control, the dry line profile as a part of the over all cross machine direction paper profile control system. No closed loop experiences in the machine direction nor in the cross machine direction are reported. The results in [5] are analysis oriented, and no closed loop experiments are presented.

Our work concerns the detection and control of the position of the dry line in the machine direction, especially during grade changes. The dry line detection system is based on [6] with some hardware changes and further development. The slice opening is used as an actuator for the control of the dry line position. Control of the dry line contour is not treated in this work.

This work is one part of a larger joint project with our industrial cooperator AssiDomän Kraftliner in Piteå, Sweden. The aim of the project is to increase the degree of automation of the wet end, especially during grade changes. The decision to control the dry line machine direction position was based upon extensive interviews with the machine operators and other personnel to find the main problem areas on the paper machine.

The novelty of this work lies in that we have implemented a system for measurement and control of the dry line machine direction position, which have been evaluated on an paper machine in production by performing

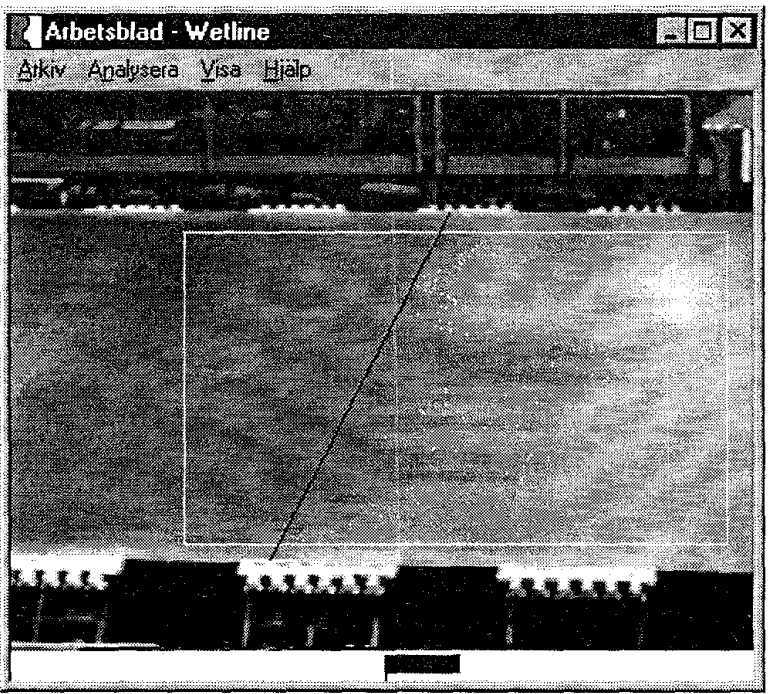

Figure 2: The analyzing area, reference line and the enhanced dry line are superimposed over the video image, and the error bar is placed below. Notice the wet streaks.

both open loop experiments and closed loop experiments during grade changes.

The rest of the article is organized as follows. Section two treats the system for detection of the dry line, section three concerns the open loop experiments and the necessary modeling, and in section four the closed loop results are presented. Some conclusions are presented in section five.

\section{Dry line detection}

The hardware for dry line detection used in this project consists of a small monochrome 1/2" CCD camera (JAI M50) with the resolution of $752 \times 582$ pixels with an appropriate lens such that the field of vision covers the essential parts of the wire, see figure 1 and 2. As frame grabber we are using a Matrox Meteor mounted in a PC. The $\mathrm{PC}$ is communicating with the control system via a RS232 serial link.

The calculation of the dry line consists of several steps. First a normalized histogram of the grayscales is calculated for a selected area of the frame as

$$
p_{i}=b_{i}\left(\sum_{j \in G} b_{j}\right)^{-1}
$$

were $b_{i}$ is the number of pixels with graylevel $i$ and $G$ is the set of actual graylevels. The area that is analyzed is easily selected by the operator such that it covers only the appropriate parts of the wire. To find the dry line we 
set up a hypothesis that the graylevels of the picture can be classified into two classes. Class $A$ with low values and class $B$ with high levels. The task is then to find the graylevel that "best" separates the two classes. Our approach is to define the interclass variance as

$$
\sigma_{M}^{2}(k)=\omega_{A}\left(\mu_{A}-\mu(G)\right)^{2}+\omega_{B}\left(\mu_{B}-\mu(G)\right)^{2}
$$

were $\omega$ is the cardinality and $\mu$ is the mean value for a class, both depending on the dividing level $k$, and the mean value for the whole set. Then

$$
\eta(k)=\frac{\sigma_{M}^{2}(k)}{\sigma_{\text {tot }}^{2}}
$$

is one of many possible measures of class separability, see more in [3]. Maximizing $\eta(k)$ with respect to $k$ gives the optimal value of $k$ to separate the classes. The cardinality of class A and $B$ respectively is

$$
\omega_{A}=\sum_{i=1}^{k} p_{i} \triangleq \omega(k)
$$

and $\omega_{B}=1-\omega_{A}$. The mean values of the classes are then

$$
\mu_{A}=\sum_{i=1}^{k} i \frac{p_{i}}{\omega_{A}}=\frac{1}{\omega(k) \sum_{i=1}^{k} i p_{i}} \triangleq \frac{\mu(k)}{\omega(k)}
$$

and

$$
\mu_{B}=\frac{\mu(G)-\mu(k)}{1-\omega(k)}
$$

respectively. By substituting (2) and (4) - (6) into (3) we get the optimal value as

$$
k_{o p t}=\arg \max _{k} \frac{(\omega(k) \mu(G)-\mu(k))^{2}}{\omega(k)(1-\omega(k))}
$$

In the next step, each line is scanned from bright, where the intensity $I>k_{\text {opt }}$, to dark searching for the position where the intensity drops below $k_{o p t}$ and thus indicates the position of the dry line. To avoid erratic behavior in case of disturbances and odd reflections it is assured that the intensity stays below $k_{\text {opt }}$ for at least $N$ pixels, were $N$ is a tuning parameter.

All calculations so far are made in the coordinate system of the camera. Since the camera can be mounted in an non-orthogonal position relative to the wire, we have to apply a nonlinear transformation to be able to calculate the mean deviation of the dry line expressed in distance from a given reference line on the wire, see figure 2. The actual parameters of the transformation are calculated in a calibration procedure after the camera is mounted. The procedure is semi-automatic as the operator has to mark four known positions on the wire.

The mean deviation of the dry line is low-pass filtered before it is sent to the control system.
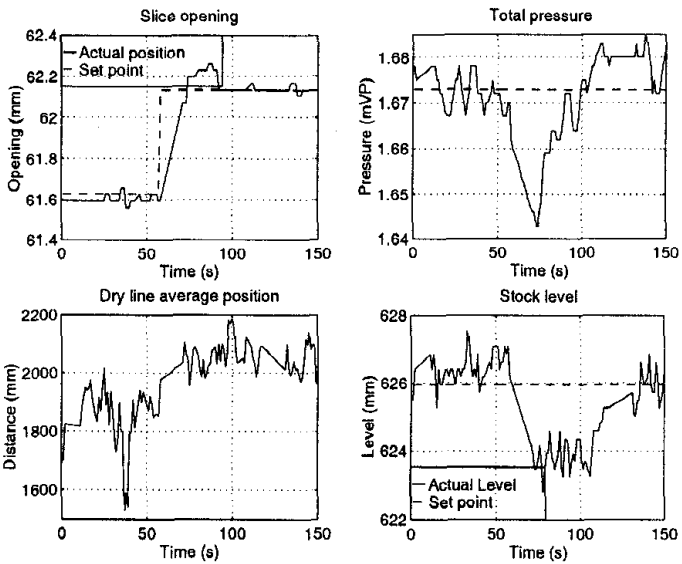

Figure 3: Slice opening, dry line position, headbox total pressure and headbox stock level during a step in the slice opening setpoint.

The frame grabber has a theoretical throughput of 30 frames/sec and works without interfering with the CPU. Thus the limiting factor of the detection is the computational speed of the PC, and with the present hardware (Pentium $60 \mathrm{MHz}$ ) new values of the dry line average position are calculated 6 to 10 times/sec. depending on the size of the analyzed area.

The ambient lighting can cause problem with specular reflections, thus it is very important that the lighting is carefully arranged. For examples of possible solutions of the lighting arrangements see [6] and [11].

\section{Open loop experiments and modelling}

The dry line model presented here is simple but adequate enough for control design. The model is based on open loop experiments with step formed changes in slice opening setpoint. Dry line movement caused by other factors than the slice opening are seen as immeasurable disturbances. Figure 3 shows the system behavior when the slice setpoint is increased stepwise. The opening of the slice is controlled by a relay controller with a dead zone, $z$ in figure 4 , of $\pm 0.1 \mathrm{~mm}$. The actuators are set screws with constant speed motors, $H(s)$ in figure 4 . The existing control of the slice opening is far from perfect and is for simplicity, and with reasonable accuracy, approximated with a second order system.

The dry line movement, $V(s)$ in figure 4 , can be considered to be directly proportional to the slice movement except for a transport delay which in this case is negligible. Preliminary experiments at different production grades show that the gain of $V(s)$ vary between 200 at 


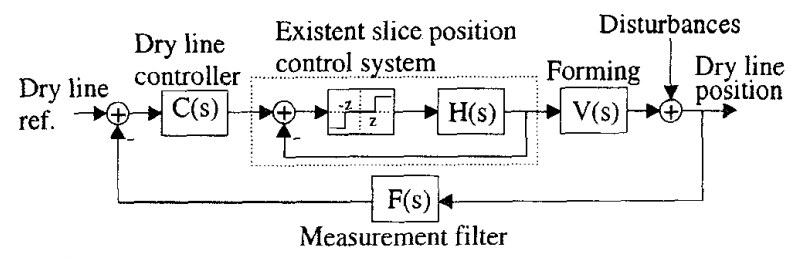

Figure 4: Block diagram of the dry line control

heavy grades and 400 at light grades. There is a tendency that the changes in dry line position are quantified by different hydrodynamic devices, see figure 2 . This ef fect is not modeled but it can lead to instability of the closed loop system if it is not considered in the design of high performance controllers.

It is the headbox volume outflow that primarily affects the dry line position, and the volume outflow is proportional to the slice opening and also proportional to the square root of the headbox total pressure at the slice. This is clearly seen from the volume flow equation (8)

$$
q=a b v=a b \sqrt{2 g h}
$$

where $a$ is the slice opening, $b$ is the width of the slice, $v$ is the outlet jet velocity, $g$ is the gravitational constant and $h$ is the headbox total pressure at the slice (in $\mathrm{m} \mathrm{H}_{2} \mathrm{O}$ ). One of the reasons for the changes in gain with different grades is, as can be seen from (8), that the total pressure is low at heavy grades and high at light grades. Another reason is that changes in wire velocity gives rise to changes in wire drying capacity, where low velocity results in high drying capacity and vice versa. An abrupt change in the slice position causes severe disturbances in the headbox total pressure and stock level, see figure 3 . This gives rise to undesirable dynamic effects, but these effects are negligible if the slice opening changes are small and slow.

\section{Closed loop experiments}

The closed loop control results presented here are preliminary results and improvements are to be expected. The results may not be good from a control point of view, but from a paper making point of view these results are very good. The main issue, at the moment, is safety, i.e. limits on the dry line position, so perfect tracking is not necessary. The potentials of this new measurement and control loop, in terms of improved over all paper machine control, are yet to be exploited.

Closed loop control experiments have been performed both during steady state operation, figure 5 , and during grade changes, figure 6 . The origin of disturbances during steady state operation is mainly the variations
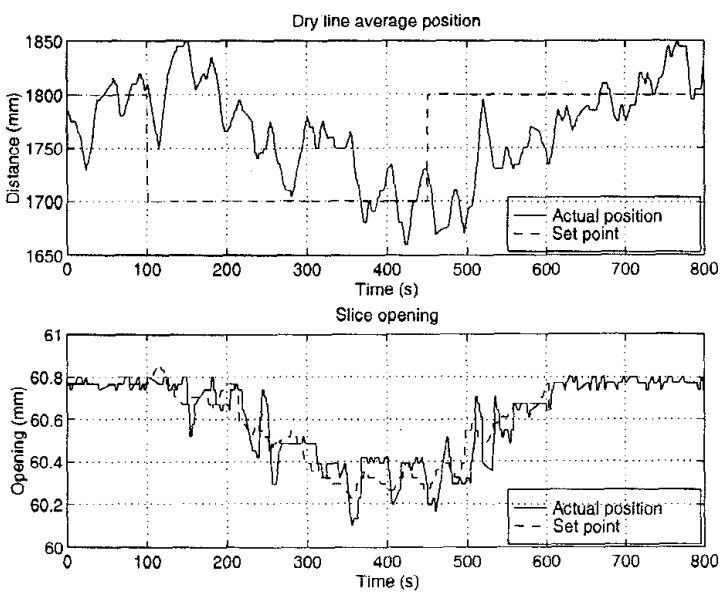

Figure 5: Dry line position and slice opening during a step in the dry line setpoint.

in the headbox total pressure. Variation in the negative pressure of the suction boxes are also a probable cause, but experiments verifying this have not been performed. The disturbances caused by the total pressure are relatively fast, and usually with a frequency content above $0.02 \mathrm{~Hz}$, see [7]. The slow disturbances appearing during steady state operation are mainly due to changes in the beating degree of the fibres, fiber mix changes and other chemical changes. According to the operators, the effect on the dry line caused by these changes is very slow, but clearly observable, typically with time constants over 5 minutes. The wear of the wire affects the drainage, which in turn affects the dry line position but this factor is extremely slow in its variation. During grade changes, disturbances caused by the ramp formed change in headbox total pressure and wire velocity, are added to the above mentioned disturbance sources. These disturbances are considered to be slow with a typical rate of change of $4 \cdot 10^{-3} \mathrm{~m} / \mathrm{s}^{2}$ in the wire velocity and $3 \cdot 10^{-3} \mathrm{~m} \mathrm{H}_{2} \mathrm{O} / \mathrm{s}$ in total pressure.

The controller and the measurement filter have to be designed so that they can handle slow dry line position variations and have low sensitivity to fast variations. The limitations in speed and the nonlinearity of the slice control, and the coupling between total pressure and changes in slice opening could cause instability if the controller is designed to be too sensitive to fast disturbances. Initial values for the filter were chosen, and an PI controller was initially designed so that the added pole would slow down the closed loop system. The initial design was implemented in the machine control system and the controller and filter parameters were manually adjusted during initial experiments. Figure 5 shows the closed loop behavior when the slice position setpoint is changed stepwise, and it can be seen that the 

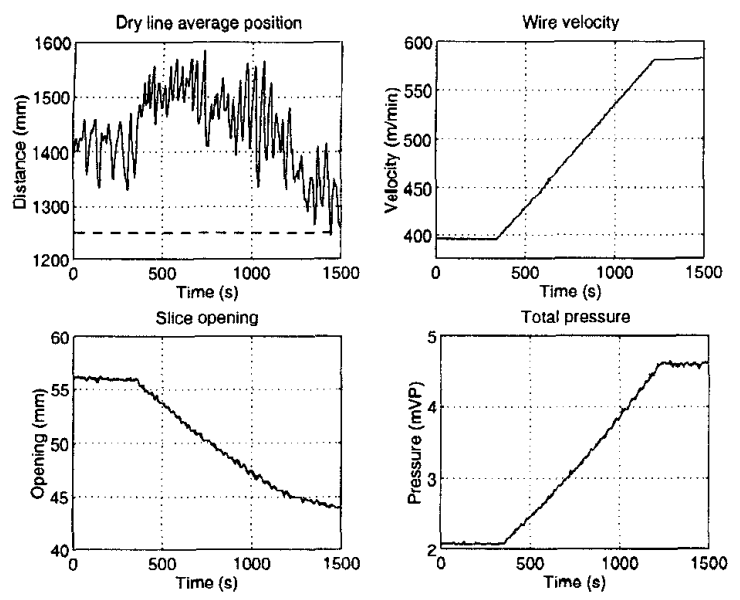

Figure 6: Dry line, slice opening, wire velocity and headbox total pressure during a grade change.

system behaves as expected. The shortcomings of the slice positioning system can be seen in its poor set point tracking properties. The main goal of this work was to control the position of the dry line during grade changes and an example of this is shown in figure 6 . There is, as can be expected with a PI controller, a constant error due to the ramp disturbances, i.e., the changes in wire velocity and total pressure. The initial error, up to $400 s$ is caused by an earlier change in beating degree of the fibres. During a grade change the whole wet end system gets disturbed and the system might not settle until a long time after the actual grade change has finished, thus causing disturbances on the dry line for a long time.

\section{Conclusions}

We have in this work presented an implementation of a dry line positioning system that is practically useful. The main problem, from a practical point of view, is the light arrangements. This problem, which is application specific, requires a thorough analysis of the light conditions. The PI controller used in the closed loop experiments, and whose design was based on a relatively simple model, proved to be sufficient for control of the dry line position during grade changes. The dry line detection system has been tested on the AssiDomän Kraftliner plant in Piteå, Sweden, and its function has been positively verified by the machine operators for an extensive period of time. The introduction of automatic control of the dry line position has been welcomed by the operators because it is a demanding manual control task during grade changes. Further development of the control system will be to improve the slice positioning system, redesign of the dry line controller with robust and/or gain scheduling methods, feedforward of wire velocity or headbox total pressure to improve the tracking capabilities and development of error models.

\section{Acknowledgments}

The authors are grateful for the support from Stefan Rönnbäck at AssiDomän Corporate R\&D in Piteå, Professor Pertti Mäkilä at Luleå University of technology, professor Antti Niemi at Helsinki University of Technology and, last but not least, the plant personnel at AssiDomän Kraftliner in Piteå, Sweden, for letting us work on their paper machine. Partial financial support to the authors from the Swedish National Board for Industrial and Technical Development is gratefully acknowledged.

\section{References}

[1] Bialkowki W. L., US Pat. No. 4,500,968, 1985.

[2] Burrows P. R. M., Raymond I. M., Terry M. P. A., UK Pat No. 1360992, 1974.

[3] Fukunaga K, Statistical Pattern Recognition, Academic Press, London, 1990.

[4] Heaven E. M., et al, Recent advances in cross machine profile control using modern estimation and control technology 2:nd IEEE Conf. on Contrl. App., Vancouver B.C., 1993.

[5] Kjaer A. P., Wellstead P. E., Heath W. P., Detection and analysis of dry-line on paper machines, IFAC 13th Tri. W. Con., San Francisco, USA, 1996.

[6] Käll L., Morén U., Measurement of wet end dewatering capacity with digital image processing, in Swedish, Master Thesis, Lulea University of Technology, January 1995

[7] Larsson J E, Olsson T, On the control of headbox outlet jet velocity, in Swedish, Master Thesis, Lulcå University of Technology, April 1995.

[8] Larsson J E, Olsson T, Vidgren L, Headbox model correction with a laser based velocity meter, Proc. Mechatronics ' 96 conf., Universidade do Minho Guimarães, Portugal, 1996.

[9] Nader A., Lebeau B., Gauthier J. P., Foulard C., Rarnaz A., New Developments in Multivariable Control of $\mathrm{Pa}$ per Machine Head boxes, Int.Symp. on Paper Machine Headboxes, Montreal, 1979.

[10] Niemi A. J., Berndtson J., Karine S., Towards faster control of paper machine by dry line measurement, International CD symposium of the XIV IMEKO, Tampere, Finland, June 1997

[11] Niemi A., Euro. Pat. No. EP 0586458B1, 1997.

[12] Wahlström B., Headbox operation, TAPPI seminar notes, 1985 wet end operations, 1985 .

[13] Öhlund U., Causes to moisture variations during grade changes, in Swedish, Master Thesis, Luleå University of Technology, December 1996. 